\title{
Modelling Length/Pressure Hysteresis of a Pneumatic Artificial Muscle using a Modified Prandtl-Ishlinskii Model
}

\author{
Yixiang Liu - Xizhe Zang* - Zhenkun Lin - Xinyu Liu - Jie Zhao \\ Harbin Institute of Technology, State Key Laboratory of Robotics and System, China
}

\begin{abstract}
Pneumatic artificial muscles have been widely used in various fields owing to their inherent compliance and high power-to-weight ratio. However, the natural hysteresis nonlinearity including length/pressure hysteresis and force/pressure hysteresis degrades their performance in precise tracking control, making it necessary to build a mathematical hysteresis model for hysteresis compensation. This paper deals with the modelling of length/pressure hysteresis of pneumatic artificial muscles. The length/pressure hysteresis loops measured by the isotonic test are found to be asymmetric and independent of the external load when the load is small. Considering that the classical Prandtl-Ishlinskii model is only effective for symmetric hysteresis, a modified Prandtl-Ishlinskii model is proposed to describe the length/pressure hysteresis behaviour. The developed model utilizes two asymmetric operators with simple mathematical forms to independently model the ascending branch and descending branch of hysteresis loops. The model parameters are identified using the recursive least square algorithm. Comparisons between simulation results and experimental measurements demonstrate that the proposed model can characterize the asymmetric major hysteresis loop and minor hysteresis loops with high accuracy.

Keywords: asymmetric hysteresis, length/pressure hysteresis, modified Prandtl-Ishlinskii model, pneumatic artificial muscles, recursive least square algorithm
Highlights
- $\quad$ The length/pressure hysteresis of a single pneumatic artificial muscle is found to be asymmetric and independent of small external load through experimental measurements. characterize the length/pressure hysteresis of the pneumatic artificial muscle.
- $\quad$ The parameters of the modified Prandtl-Ishlinskii model is identified quite conveniently using the recursive least mean algorithm.
- $\quad$ The proposed model has high accuracy in describing the major hysteresis loop and minor hysteresis loops.

- This paper proposes a modified Prandtl-Ishlinskii model composing of two independent asymmetric play operators to
\end{abstract}

\section{INTRODUCTION}

Pneumatic artificial muscle (PAM) is a relatively new kind of elastic pneumatic actuator. Simply put, PAM is a cylindrical rubber bladder surrounded by a braided fibre sheath, with both ends closed [1]. When supplied with compressed gas, the bladder experiences radial expansion, consequently generating axial contraction and exerting a pulling force on an external load [2]. Owing to its particular structure and materials, PAM has several unique advantages compared to hydraulic and electric actuators, for instance, high power-toweight ratio, compact size, inherent compliance, as well as low cost [3] and [4]. Therefore, PAMs are increasingly used in industrial applications [5] to [7], bionic robots [8] to [10], surgical instruments [11], rehabilitation devices [12] to [14], and so forth.

For a PAM, the input is internal pressure, while the outputs are contraction length and pulling force. In other words, the contraction length and pulling force depend on the internal pressure. Thus, the position or force control of PAM is based on the control of internal pressure. However, the relationships between the length and internal pressure in the pressurizing and depressurizing processes are different. The same are true for the relationships between the force and internal pressure. These differences are called length/ pressure hysteresis and force/pressure hysteresis, respectively, and are caused by the elasticity of the bladder, and the friction between the inner tube and the outer sheath [15]. The hysteretic behaviour increases system nonlinearity and makes it more difficult to achieve precise position or force tracking control. To deal with this problem, one possible solution is to build a mathematical hysteresis model and utilize its inversion for hysteresis compensation to obtain the approximately linear response of PAM.

To date, many investigations of the hysteresis modelling of PAM have been made. Van Damme et al. [16] studied the hysteresis behaviour of pleated PAM, and found that the dimensionless force was independent of gauge pressure and contraction rate. A Preisach-based hysteresis model was built for the force/contraction characteristics, which only performed well in the contraction range from $7 \%$ to $20 \%$. Through investigation, Vo-Minh et al. [17] to [19] found the pressure/length hysteresis and the force/ length hysteresis [20] were quasi-rate independent 
and history dependent, and could be described by the Maxwell-slip model. Then they further extended their work to model the torque/angle hysteresis of a joint driven by PAMs in antagonistic configuration, using the model derived for individual PAMs [21]. Frank Schreiber adopted the classical Preisach model to describe the static hysteresis behaviour of a manipulator driven by antagonistic PAMs and then used its inversion for feedforward hysteresis compensation [22] and [23]. Kosaki and Sano [24] also constructed the contraction/pressure hysteresis model in the form of the Preisach model for compensation of a parallel manipulator driven by three PAMs. Ito et al. [25] modelled the length/pressure hysteresis of a PAM at a certain external load using a stop model, which was a function of the contraction ratio and applied load. Lin et al. [26] investigated pressure/length hysteresis characteristics for a dual PAM system using three different models, including a Prandtl-Ishlinskii model, a Bouc-Wen model, and a Maxwell-slip model. Comparisons indicated that the former two models were more effective than the third one.

Among the above studies, the Preisach model is the most widely used because of its applicability in various hysteretic actuators, such as electromagnetic actuators [27], piezoceramic actuators [28] and [29], and magnetostrictive actuators [30]. However, its mathematical form is highly complex, making it difficult to derive the analytic inverse model. In contrast, the Prandtl-Ishlinskii model has simple play operators, which facilitate calculation of the inverse hysteresis model [31] and [32]. Nevertheless, it is not applicable to modelling asymmetric hysteresis behaviour, owing to the symmetric property of the classical play operators [33] and [34].

This paper is mainly intended for the modelling of the asymmetric length/pressure hysteresis of PAM, which is essential for position tracking control. Based on the classical Prandtl-Ishlinskii model, a modified Prandtl-Ishlinskii (MPI) model is proposed by replacing the classical play operators with more flexible elementary operators. This model utilizes two independent operators to describe the ascending branch and descending branch of hysteresis loops respectively. In addition, the model parameters can be identified using a recursive least square algorithm within several steps.

The remaining content of this paper is organized as follows. First, the isotonic experiment setup for measuring the length/pressure hysteresis of PAM is presented. Then the MPI model is introduced in detail. After that, the MPI model is identified and verified using experiment data. Finally, some conclusions and discussion points for future work are given.

\section{LENGTH/PRESSURE HYSTERESIS OF PNEUMATIC ARTIFICIAL MUSCLES}

\subsection{Experimental Apparatus}

In this study, an isotonic experiment is carried out to measure the relationships between the contraction, internal pressure, and an external load of a PAM [35]. Currently, there are several PAM manufacturers including FESTO located in Germany, Shadow in the UK, and Bridgestone and HITACHI in Japan. Among them, the PAMs from FESTO are the cheapest and the most readily available. Thus, a commercial PAM from FESTO (DMSP-20-180) is adopted in the experiment, as illustrated in Fig. 1. The inner diameter and nominal length of the contractable membrane are $20 \mathrm{~mm}$ and $180 \mathrm{~mm}$ respectively. The maximum permitted contraction ratio of the PAM is about $25 \%$ under the maximum operating pressure of $0.6 \mathrm{MPa}$.

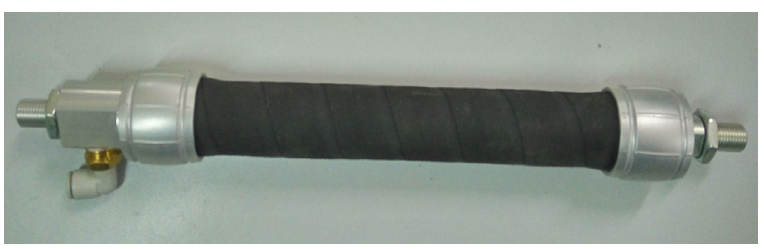

Fig. 1. A commercial PAM manufactured by FESTO

Fig. 2 presents the schematic and experimental physical apparatus. The upper end of the PAM is fastened on the fixed frame, and the lower end is mounted with an external load. Compressed air is supplied to the PAM through a proportional pressure regulator. The internal pressure and the contraction of the PAM are measured by a pressure transducer and a linear displacement transducer, respectively. The computer sends the operating voltage to the proportional pressure regulator and receives the measurement signals through the $\mathrm{D} / \mathrm{A}$ and $\mathrm{A} / \mathrm{D}$ converters. The outputs of all transducers are recorded on the computer for further analysis.

\subsection{Measurement of Length/Pressure Hysteresis}

The length/pressure hysteresis of a single PAM was measured using the above experimental apparatus. To reduce the impact of the acceleration of the applied load, the experiment was carried out under the assumption of a quasi-static process. The process of the experiment is as follows. In the initial state, 

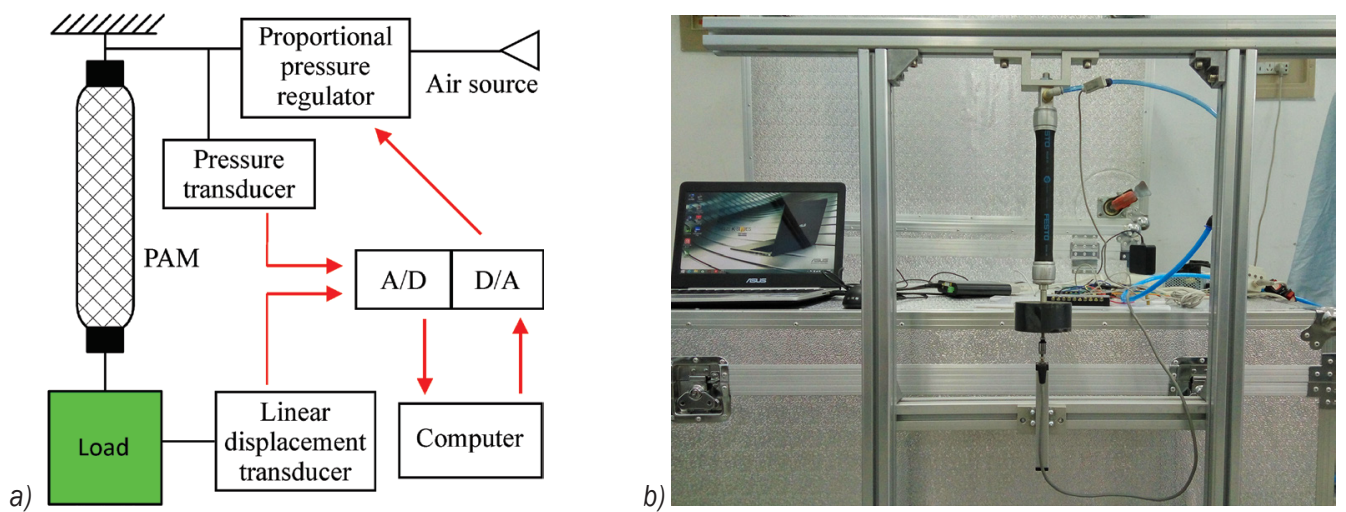

Fig. 2. Experimental apparatus; a) schematic experimental apparatus, b) physical experimental apparatus

the gauge pressure inside the PAM was zero, and the PAM was at its full length with a certain load hanging below. Then the PAM was pressurized and depressurized following a reference pressure signal in the form of triangle-wave with various amplitudes. The amplitudes decreased from $0.6 \mathrm{MPa}$ to 0.2 $\mathrm{MPa}$ at a constant interval of $0.1 \mathrm{MPa}$. Fig. 3 shows the reference pressure signal. During this process, the internal pressure and contraction of PAM were

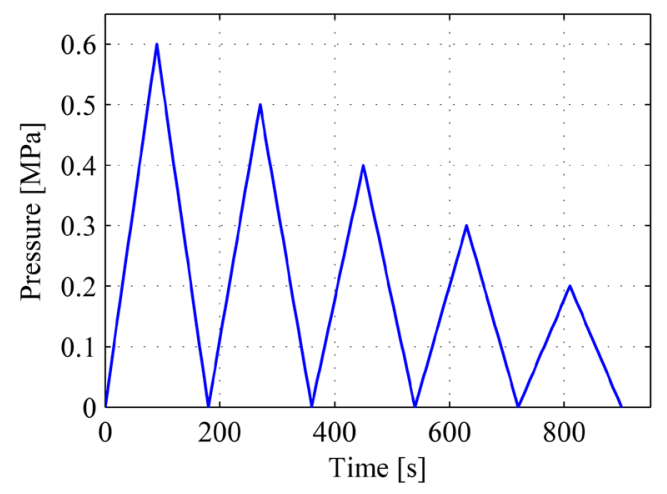

Fig. 3. The reference pressure signal

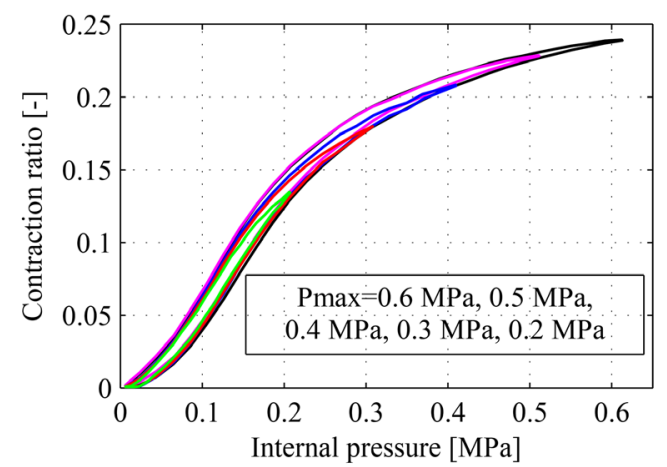

Fig. 4. Relationship between the contraction ratio and internal pressure in the case of $1 \mathrm{~kg}$ load measured and recorded. To obtain the relationships between the length/pressure hysteresis and applied external load, the above process was repeated in different cases where the applied load was $1 \mathrm{~kg}, 2 \mathrm{~kg}$, $3 \mathrm{~kg}$, and $4 \mathrm{~kg}$, respectively.

When the experiment was completed, the measured data were plotted and then analysed. Figs. 4 to 7 show the relationships between the contraction ratio and internal pressure of the PAM when the

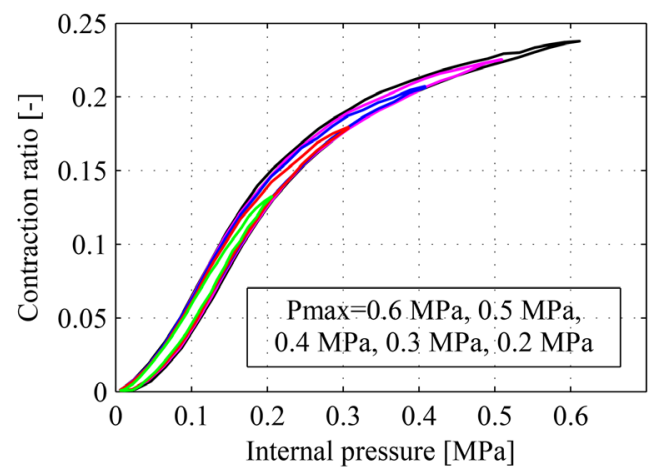

Fig. 5. Relationship between the contraction ratio and internal pressure in the case of $2 \mathrm{~kg}$ load

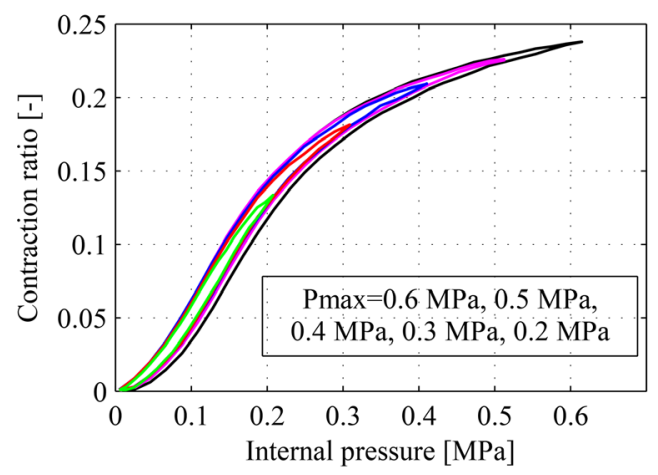

Fig. 6. Relationship between the contraction ratio and internal pressure in the case of $3 \mathrm{~kg}$ load 
external load is $1 \mathrm{~kg}, 2 \mathrm{~kg}, 3 \mathrm{~kg}$, and $4 \mathrm{~kg}$, respectively. The contraction ratio $\varepsilon$ is calculated using the following equation:

$$
\varepsilon=\frac{\Delta L}{L}
$$

where $\Delta L$ is the measured contraction, and $L$ is the nominal length of the PAM.

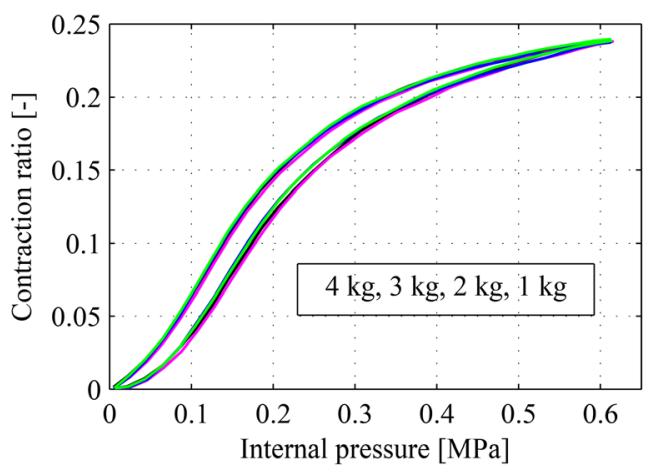

Fig. 7. Relationship between the contraction ratio and internal pressure in the case of $4 \mathrm{~kg}$ load

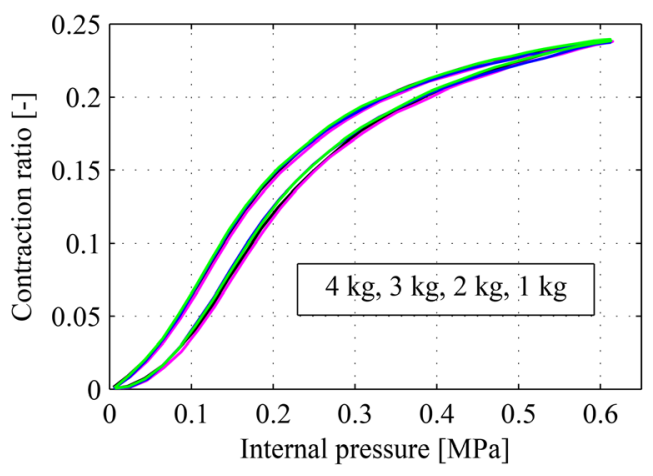

Fig. 8. The major length/pressure hysteresis loops with different loads

According to these four figures, several findings can be suggested as follows. First, hysteresis loops including the major loop and minor loops exist between the contraction ratio and internal pressure of PAM. The hysteresis loop formed under the maximum input pressure is termed the major loop. The loops inside the major loop are termed minor loops, which are formed when the input extrema are less than the maximum value. Second, all the hysteresis loops are asymmetric.

Fig. 8 presents the major length/pressure hysteresis loops with different loads. It is quite clear that these major loops almost coincide. Then the third finding can be obtained, i.e. when the external load is small, the length/pressure hysteresis is not dependent on the external load.

\section{LENGTH/PRESSURE HYSTERESIS MODELLING}

\subsection{The Classical PrandtI-Ishlinskii Model}

The classical Prandtl-Ishlinskii hysteresis model uses the summation of a finite number of weighted backlash operators with different thresholds to characterize hysteresis behaviour in the continuous space, as presented in Fig. 9. Analytically, suppose $C_{\mathrm{m}}$ represents the space of the piecewise monotone and continuous functions, and $0=t_{0}<t_{1}<\cdots<t_{\mathrm{N}}=t_{\mathrm{E}}$ are subintervals in $\left[0, t_{\mathrm{E}}\right]$. For the input $u(t) \in C_{\mathrm{m}}\left[0, t_{\mathrm{E}}\right]$, which is monotone on each of the subintervals $\left[t_{i}, t_{i+1}\right]$ for $i=0,1,2, \ldots, N-1$, the backlash operator $H_{\mathrm{r}}\left[u, w_{0}\right](t)$ can be defined as [36] and [37]:

$$
\begin{aligned}
w(t) & =H_{r}\left[u, w_{0}\right](t)= \\
& =\max \left\{u(t)-r, \min \left\{u(t), w\left(t_{i}\right)\right\},\right.
\end{aligned}
$$

where $w(t)$ is the output of the backlash operator, $r$ is the threshold value, and $w_{0}$ is the initial condition.

According to the initial consistency condition, it will have:

$$
w(0)=\max \left\{u(0)-r, \min \left\{u(0), w_{0}\right\}\right\} .
$$

The input-output relationship of the above backlash operator is shown in Fig. 10. Then the output of the hysteresis model can be written as:

$$
y(t)=p u(t)+\int_{0}^{R} q \cdot H_{r}\left[u, w_{0}\right](t) d r,
$$

where $p$ is a constant parameter, and $q$ is the density function that needs to be identified from the experimental data.

To facilitate calculation and application of the hysteresis model in computer programming, it is necessary to approximate the integral using summation. Thus, the classical Prandtl-Ishlinskii hysteresis model in the discrete form can be expressed as:

$$
y(t)=p u(t)+\sum_{i=1}^{n} q_{i} H_{r i}\left[u, w_{0}\right](t),
$$

with $n$ representing the number of backlash operators.

Research has shown that the classical PrandtlIshlinskii model only works well for characterizing symmetric hysteresis loops. Then its viability and effectiveness are checked using the experimental data. The model validation result is shown in Fig. 11, by comparing the measured length/pressure hysteresis loop with the simulated hysteresis loop. Obviously, the classical Prandtl-Ishlinskii model cannot describe the hysteresis behaviour of the PAM. 


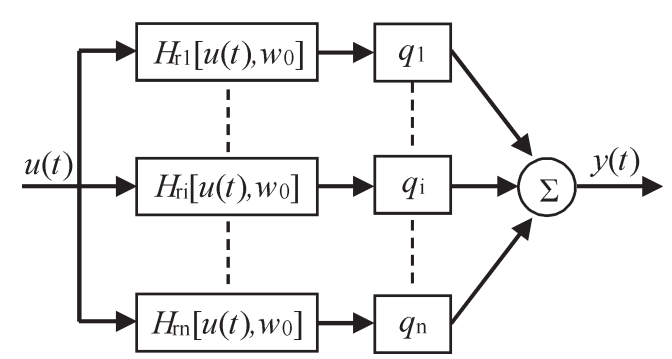

Fig. 9. Block diagram of the classical Prandtl-Ishlinskii model

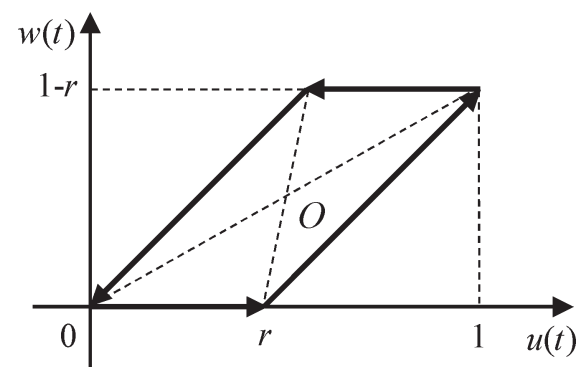

Fig. 10. Input-output relationship of the backlash operator

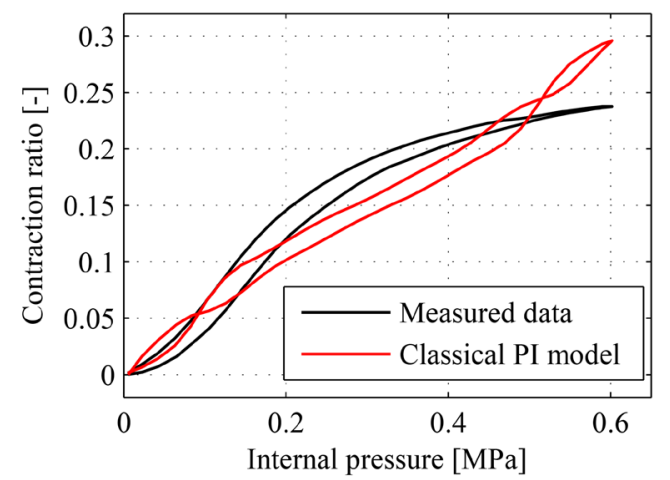

Fig. 11. Model validation result of classic Prandtl-Ishlinskii model

\subsection{The Modified PrandtI-Ishlinskii Model}

Since the classical Prandtl-Ishlinskii model is only valid for symmetric hysteresis description, a new MPI model is proposed to characterize the asymmetric length/pressure hysteresis of the pneumatic artificial muscle. According to the changes of input signals, a whole hysteresis loop can be divided into two parts: ascending branch for input increase and descending branch for input decrease. Therefore, the MPI model is also based on two independent asymmetric play operators: the play operator of the ascending branch and the play operator of the descending branch. The two play operators describe the ascending hysteretic nonlinearity and descending hysteretic nonlinearity of the system, respectively. Moreover, their combination is capable of modelling the full hysteresis loop.
For any piecewise monotone input function $u(t) \in C_{\mathrm{m}}\left[0, t_{\mathrm{E}}\right] \rightarrow[0,1]$, the elementary play operator of the ascending branch is defined as:

$$
H_{r}^{a}\left[u, w_{0}\right](t)=\left\{\begin{array}{ll}
w_{i-1} & \Delta u \geq 0, u_{i} \leq r \\
\frac{1+r}{1-r}\left(u_{i}-r\right) & \Delta u \geq 0, r<u_{i} \leq \frac{1+r}{2} \\
u_{i} & \Delta u \geq 0, u_{i}>\frac{1+r}{2} \\
u_{i} & \Delta u<0
\end{array} .\right.
$$

Similarly, the elementary play operator of the descending branch is defined as:

$$
H_{r}^{d}\left[u, w_{0}\right](t)=\left\{\begin{array}{ll}
f\left(u_{m}\right) & \Delta u \geq 0 \\
u_{i} & \Delta u<0, \frac{1-r}{2} \leq u_{i}<1-r \\
\frac{1+r}{1-r} u_{i}-r & \Delta u<0, u_{i} \geq 1-r \\
u_{i} & \Delta u<0
\end{array} .\right.
$$

In the above equation, $f\left(u_{m}\right)$ is the function of the dominant extrema of the input um, which is described by a quadratic polynomial, expressed as:

$$
f\left(u_{m}\right)=c_{0}+c_{1} \cdot u_{m}+c_{2} \cdot u_{m}{ }^{2} .
$$

For the elementary play operators, the threshold values $r$ are distributed within the range not exceeding the maximum amplitude of the input, i.e., $0 \leq r<1$. Figs. 12 and 13 present the input-output relationship of the play operator of the ascending branch and of the descending branch respectively.

Calculating the weighted sum of the two play operators, the output of the modified hysteresis model can be written as:

$$
\begin{aligned}
y(t)= & p u(t)+\sum_{i=1}^{n} q_{i}^{a} H_{r i}^{a}\left[u, w_{0}\right](t)+ \\
& +\sum_{i=1}^{n} q_{i}^{d} H_{r i}^{d}\left[u, w_{0}\right](t),
\end{aligned}
$$

where $q_{i}^{a}$ is the density function of the play operator of the ascending branch, and $q_{i}^{d}$ is the density function of the play operator of the descending branch.

The two play operators are subject to the following constraints:

$$
\left\{\begin{array}{l}
H_{r i}^{a}\left[1, w_{0}\right](t)=1 \\
H_{r i}^{d}\left[1, w_{0}\right](t)=1 \\
p+\sum_{i=1}^{n} q_{i}^{a} H_{r i}^{a}\left[1, w_{0}\right](t)+\sum_{i=1}^{n} q_{i}^{d} H_{r i}^{d}\left[1, w_{0}\right](t)=1
\end{array} .\right.
$$


Substituting Eq. (10) into Eq. (9), the output of the hysteresis model can be rewritten as:

$$
\begin{aligned}
y(t) & =u(t)+\sum_{i=1}^{n} q_{i}^{a}\left(H_{r i}^{a}\left[u, w_{0}\right](t)-u(t)\right)+ \\
& +\sum_{i=1}^{n} q_{i}^{d}\left(H_{r i}^{d}\left[u, w_{0}\right](t)-u(t)\right) .
\end{aligned}
$$

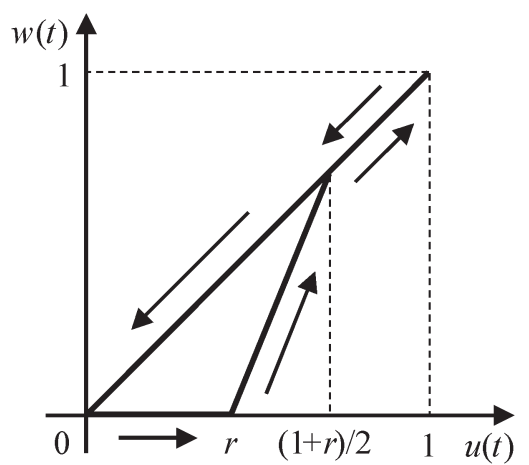

Fig. 12. The elementary play operator of the ascending branch

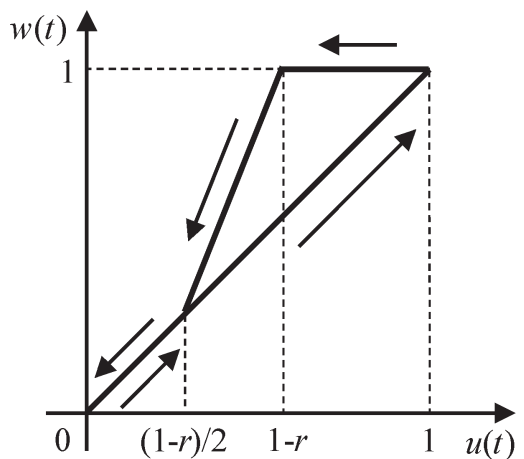

Fig. 13. The elementary play operator of the descending branch

\section{MODEL IDENTIFICATION AND VALIDATION}

In the MPI model, there are a total of $2 n+3$ parameters that need to be identified. They are the weightings $q_{i}^{a}(i=1,2, \cdots, n)$ of the play operators of the ascending branch, the weightings $q_{i}^{d}(i=1,2, \cdots, n)$ of the play operators of the descending branch, and the coefficients $c_{\mathrm{i}}(i=1,2,3)$ of the quadratic polynomial function $f\left(u_{m}\right)$. The input and output of the MPI model are constrained within the range $[0,1]$. Therefore, the measured data of the isotonic experiment, i.e., the internal pressure and the contraction ratio denoted as $\left(x_{\mathrm{i}}, y_{\mathrm{i}}\right)$ need to be normalized from the physical space to the model space to satisfy the constraints first before they can be used for the identification of model parameters [38]. When the identification is completed, the outputs of the hysteresis model are transformed back to the physical space. The normalization of measured data from the physical space to the model space is defined as:

$$
\left\{\begin{array}{l}
x_{n i}=\frac{x_{i}-x_{\min }}{x_{\max }-x_{\min }} \\
y_{n i}=\frac{y_{i}-y_{\min }}{y_{\max }-y_{\min }}
\end{array} .\right.
$$

The thresholds $r$ of the elementary play operators are predefined as:

$$
r_{i}=\frac{i-1}{n} \quad 1 \leq i \leq n .
$$

The whole identification process can be divided into two steps. First, the weightings of the elementary play operators are identified with the measured data of the major hysteresis loop. After that, the coefficients of the quadratic polynomial function are calculated with the measured data of the minor hysteresis loops. The model parameters are identified using a recursive least squares (RLS) algorithm, which is one of the most well-known algorithms used in signal processing, parameters estimation and system control which recursively finds the coefficients minimizing a weighted linear least squares cost function relating to the training data. It has several unique advantages, such as fast convergence rate, easy programming, and avoiding the eigenvalue spread problem. RLS algorithm works as follows: when the observations of the system parameters are obtained, the new observations are updated by adding a correction to the old values, and then the process iterates until the observations reach acceptable accuracy. The parameter iteration law is given by:

$$
\left\{\begin{array}{l}
K(k)=\frac{P(k-1) \cdot \phi^{T}(k-1)}{1+\phi(k-1) \cdot P(k-1) \cdot \phi^{T}(k-1)} \\
P(k)=[I-K(k) \cdot \phi(k-1)] \cdot P(k-1) \\
q(k)=q(k-1)+K(k) \cdot[y(k)-\phi(k-1) \cdot q(k-1)]
\end{array}\right.
$$

with $q(k)$ representing the observations of the $k^{\text {th }}$ estimation. The iteration law ensures that the parameters will converge to stable values after several steps of iteration.

The number of play operators determines the accuracy of hysteresis modelling. More play operators will guarantee higher accuracy but demand heavier computation. Fig. 14 shows the major length/pressure hysteresis loops simulated by MPI models with different numbers of play operators. It can be easily found that when the number is greater than or equal 
to 20 , the model can predict the major hysteresis loop with sufficient accuracy. Table 1 presents the identified weightings of play operators in the case in which the number is 20 . The quadratic polynomial function $f\left(u_{m}\right)$ is identified and written as:

$$
f\left(x_{m}\right)=0.5496+1.0109 x_{m}-0.5661 x_{m}{ }^{2} .
$$

Fig. 15 shows the model validation result of the MPI model. Obviously, the MPI model can describe the hysteresis behaviour of the PAM including both major loop and minor loops with very high accuracy.

Table 1. The identified weightings of play operators

\begin{tabular}{|c|c|c|c|c|c|}
\hline$r_{i}$ & $q_{i}^{a}$ & $q_{i}^{d}$ & $r_{i}$ & $q_{i}^{a}$ & $q_{i}^{d}$ \\
\hline 0 & -0.0014 & -0.0014 & 0.5 & -0.0277 & 0.0689 \\
\hline 0.05 & 0.5689 & 0.0915 & 0.55 & 0.0476 & 0.0718 \\
\hline 0.1 & 0.3541 & 0.0523 & 0.6 & -0.0141 & 0.0152 \\
\hline 0.15 & 0.3932 & -0.0811 & 0.65 & -0.0679 & 0.0989 \\
\hline 0.2 & 0.1718 & 0.0415 & 0.7 & -0.0778 & 0.0695 \\
\hline 0.25 & -0.1755 & 0.0804 & 0.75 & -0.0379 & 0.0974 \\
\hline 0.3 & -0.1351 & 0.0268 & 0.8 & 0.0116 & 0.0403 \\
\hline 0.35 & -0.2026 & 0.0013 & 0.85 & -0.0655 & 0.0481 \\
\hline 0.4 & -0.0506 & 0.0871 & 0.9 & -0.0217 & 0.0157 \\
\hline 0.45 & -0.1292 & -0.0031 & 0.95 & -0.0378 & 0.0110 \\
\hline
\end{tabular}

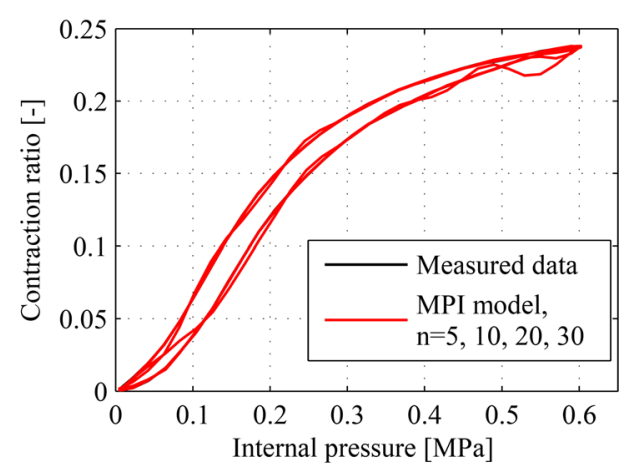

Fig. 14. Major length/pressure hysteresis loops simulated by MPI models with different numbers of play operators

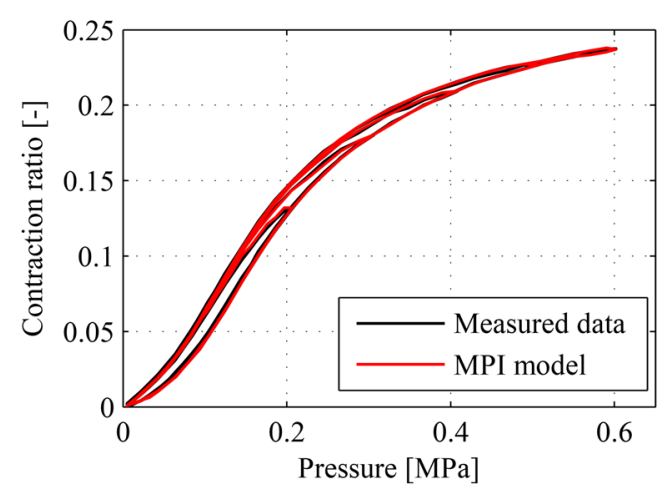

Fig. 15. Model validation result of the MPI model

\section{CONCLUSION AND DISCUSSION}

In this paper, a modified Prandtl-Ishlinskii model is proposed to model the asymmetric length/pressure hysteresis behaviour of PAMs. The proposed model is combined with two asymmetric play operators which are used to independently describe the ascending branch and descending branch of the hysteresis loops. The model parameters are identified using the recursive least square algorithm, making the identification process very simple and convenient. Compared to existing hysteresis models, the benefits of the proposed MPI model are in its relatively more concise mathematical form and its high validity and effectiveness in reproducing the asymmetric length/ pressure hysteresis including both major hysteresis loop and minor hysteresis loops. Furthermore, the MPI model can simplify the derivation of the inverse hysteresis model. In fact, the inversion of the length/pressure hysteresis can be obtained in a more straightforward way instead of mathematical computation. More precisely, taking the contraction ratio as the input and the pressure as the output, the inverse length/pressure hysteresis model can be directly identified from measurements using the proposed MPI model.

The above benefits suggest that the new MPI model can play some role in the hysteresis compensation of PAMs. Therefore, it will be interesting to study its implementation in the position control of a single PAM and/or robotic joints actuated by PAMs. In this work, only the quasi-static hysteretic behaviour of PAMs is studied because the dynamic hysteresis is too complicated to be modelled precisely. When the MPI model is applied to dynamic systems, various model uncertainties and external disturbances that will affect its effectiveness remain. However, some intelligent control methods, such as nonlinear adaptive control and robust nonlinear control can deal with the above issue. Therefore, in the future, we will focus on researching a position controller that combines the robust feedback control scheme with MPI model-based hysteresis compensation to achieve better position tracking performance of PAMs.

\section{ACKNOWLEDGEMENTS}

This research is funded and supported by the National Natural Science Foundation of China (Grant No. 51675116). 


\section{REFERENCES}

[1] Tondu, B. (2012). Modelling of the McKibben artificial muscle: A review. Journal of Intelligent Material Systems and Structures, vol. 23, no. 3, p. 225-253, Dol:10.1177/1045389X11435435.

[2] Daerden, F., Lefeber, D. (2000). Pneumatic artificial muscles: actuators for robotics and automation. European Journal of Mechanical and Environmental Engineering, vol. 47, no. 1, p. 10-21.

[3] Caldwell, D.G., Tsagarakis, N., Medrano-Cerda, G.A. (2000). Bio-mimetic actuators: polymeric pseudo muscular actuators and pneumatic muscle actuators for biological emulation. Mechatronics, vol. 10, no. 4-5, p. 499-530, Dol:10.1016/ S0957-4158(99)00071-9.

[4] Davis, S., Tsagarakis, N., Canderle, J., Caldwell, D.G. (2003). Enhanced modeling and performance in braided pneumatic muscle actuators. International Journal of Robotics Research, vol. 22, no. 3-4, p. 213-227, D0l:10.1177/02783649030220 03006.

[5] Palko, A., Smrček, J. (2011). The use of pneumatic artificial muscles in robot construction. Industrial Robot: An International Journal, vol. 38, no. 1, p. 11-19, DOl:10.1108/01439911111097805.

[6] Hošovský, A., Havran, M. (2012). Dynamic modeling of one degree of freedom pneumatic muscle-based actuator for industrial applications. Technical Gazete - Tehnički Vjesnik, vol. 19, no. 3, p. 673-681.

[7] Robinson, R.M., Kothera, C.S., Wereley, N.M. (2014). Control of a heavy-lift robotic manipulator with pneumatic artificial muscles. Actuators, vol. 3, no. 2, p. 41-65, Dol:10.3390/ act3020041.

[8] Ogawa, K., Narioka, K., Hosoda, K. (2011). Development of whole-body humanoid "Pneumat-BS" with pneumatic musculoskeletal system. Proceedings of the 2011 IEEE/RSJ International Conference on Intelligent Robots and Systems, p. 4838-4843, DOI:10.1109/IROS.2011.6095091.

[9] Liu, Y.X., Zang, X.Z., Liu, X.Y., Wang, L. (2015). Design of a biped robot actuated by pneumatic artificial muscles. Bio-Medical Materials and Engineering, vol. 26, no. s1, p. S757-S766, D0I:10.3233/bme-151367.

[10] Lei, J., Yu, H. (2014). Dynamics analysis of bionic flexible body driven by pneumatic artificial muscle for quadruped robot. Journal of Shanghai Jiaotong University, vol. 48, no. 12, p. 1688-1693. (in Chinese)

[11] Li, H.B., Kawashima, K., Tadano, K., Ganguly, S., Nakano, S. (2013). Achieving haptic perception in forceps' manipulator using pneumatic artificial muscle. IEEE/ASME Transactions on Mechatronics, vol. 18, no. 1, p. 74-85, D0l:10.1109/ TMECH.2011.2163415.

[12] Jiang, X.Z, Wang, Z.H., Zhang, C., Yang, L. (2015). Fuzzy neural network control of the rehabilitation robotic arm driven by pneumatic muscles. Industrial Robot: An International Journal, vol. 42, no. 1, p. 36-43, D0I:10.1108/IR-07-2014-0374.

[13] Huang, J., Tu, X.K., He, J.P. (2016). Design and evaluation of the RUPERT wearable upper extremity exoskeleton robot for clinical and in-home therapies. IEEE Transactions on Systems, Man, and Cybernetics: Systems, vol. 46, no. 7, p. 926-935, DOI:10.1109/TSMC.2015.2497205.
[14] Jamwal, P.K., Hussain, S., Ghayesh, M.H., Rogozina, S.V. (2016). Impedance control of an intrinsically compliant parallel ankle rehabilitation robot. IEEE Transactions on Industrial Electronics, vol. 63, no. 6, p. 3638-3647, D0l:10.1109/ TIE.2016.2521600.

[15] Tondu, B., Lopez, P. (2000). Modeling and control of McKibben artificial muscle robot actuators. IEEE Control Systems, vol. 20, no. 2, p. 15-38, Dol:10.1109/37.833638.

[16] Van Damme, M., Beyl, P., Vanderborght, B., Van Ham, R., Vanderniepen, I., Versluys, R., Daerden, F., Lefeber, D. (2008). Modeling hysteresis in pleated pneumatic artificial muscles. Proceedings of the 2008 IEEE Conference on Robotics, Automation and Mechatronics, p. 471-476, D0I:10.1109/ RAMECH.2008.4681431.

[17] Vo-Minh, T., Tjahjowidodo, T., Ramon, H., Van Brussel, H. (2009). Non-local memory hysteresis in a pneumatic artificial muscle (PAM). Proceedings of the $17^{\text {th }}$ Mediterranean Conference on Control and Automation, p. 640-645.

[18] Vo-Minh, T., Tjahjowidodo, T., Ramon, H., Van Brussel, H. (2009). Control of a pneumatic artificial muscle (PAM) with model-based hysteresis compensation. Proceedings of the IEEE/ASME International Conference on Advanced Intelligent Mechatronics, p. 1082-1087, D0l:10.1109/ AIM.2009.5229730.

[19] Vo-Minh, T., Tjahjowidodo, T., Ramon, H., Van Brussel, H. (2010). Cascade position control of a single pneumatic artificial muscle-mass system with hysteresis compensation. Mechatronics, vol. 20, no. 3, p. 402-414, D0l:/10.1016/j. mechatronics.2010.03.001.

[20] Vo-Minh, T., Tjahjowidodo, T., Ramon, H., Van Brussel, H. (2011). A new approach to modeling hysteresis in a pneumatic artificial muscle using the Maxwell-slip model. IEEE/ASME Transactions on Mechatronics, vol. 16, no. 1, p. 177-186, DOI:10.1109/TMECH.2009.2038373.

[21] Vo-Minh, T., Kamers, B., Tjahjowidodo, T., Ramon, H., Van Brussel, H. (2010). Modeling torque-angle hysteresis in a pneumatic muscle manipulator. Proceedings of the IEEE/ASME International Conference on Advanced Intelligent Mechatronics, p. 1122-1127, D0l:10.1109/ AIM.2010.5695805.

[22] Schreiber, F., Sklyarenko, Y., Schlüter, K., Schmitt, J., Rost, S., Raatz, A., Schumacher, W. (2011). Tracking control with hysteresis compensation for manipulator segments driven by pneumatic artificial muscles. Proceedings of the IEEE International Conference on Robotics and Biomimetics, $\mathrm{p}$. 2750-2755, DOI:10.1109/ROBI0.2011.6181721.

[23] Schreiber, F., Sklyarenko, Y., Runge, G., Schumacher, W. (2012). Model-based controller design for antagonistic pairs of fluidic muscles in manipulator motion control. Proceedings of the 17th International Conference on Methods and Models in Automation and Robotics, p. 499-504, D0l:10.1109/ MMAR.2012.6347868.

[24] Kosaki, T., Sano, M. (2011). Control of a parallel manipulator driven by pneumatic muscle actuators based on a hysteresis model. Journal of Environment and Engineering, vol. 6, no. 2, p. 316-327, Dol:10.1299/jee.6.316.

[25] Ito, A., Washizawa, N., Kiyoto, K., Furuya, N. (2011). Control of pneumatic actuator in consideration of hysteresis 
characteristics. Proceedings of the IEEE International Conference on Robotics and Biomimetics, p. 2541-2546, DOI:10.1109/ROBI0.2011.6181687.

[26] Lin, C.J., Lin, C.R., Yu, S.K., Chen, C.T. (2015). Hysteresis modeling and tracking control for a dual pneumatic artificial muscle system using Prandtl-Ishlinskii model. Mechatronics, vol. 28, p. 35-45, D0l:10.1016/j.mechatronics.2015.03.006.

[27] Mittal, S., Menq, C.H. (2000). Hysteresis compensation in electromagnetic actuators through Preisach model inversion. IEEE/ASME Transactions on Mechatronics, vol. 5, no. 4, p. 394-409, D0I:10.1109/3516.891051.

[28] Song, G., Zhao, J.Q., Zhou, X.Q., De Abreu-Garcia, J.A. (2005). Tracking control of a piezoceramic actuator with hysteresis compensation using inverse Preisach model. IEEE/ASME Transactions on Mechatronics, vol. 10, no. 2, p. 198-209, DOI:10.1109/TMECH.2005.844708.

[29] Xiao, S.L., Li, Y.M. (2013). Modeling and high dynamic compensating the rate-dependent hysteresis of piezoelectric actuators via a novel modified inverse Preisach model. IEEE Transactions on Control Systems Technology, vol. 21, no. 5, p. 1549-1557, DOI:10.1109/TCST.2012.2206029.

[30] Li, Z., Su, C.Y., Chai, T.Y. (2014). Compensation of hysteresis nonlinearity in magnetostrictive actuators with inverse multiplicative structure for Preisach model. IEEE Transactions on Automation Science and Engineering, vol. 11, no. 2, p. 613-619, D0I:10.1109/TASE.2013.2284437.

[31] Aschemann, H., Schindele, D. (2014). Comparison of modelbased approaches to the compensation of hysteresis in the force characteristic of pneumatic muscles. IEEE Transactions on Industrial Electronics, vol. 61, no. 7, p. 3620-3629, DOI:10.1109/TIE.2013.2287217.

[32] Janaideh, M.A.l., Rakheja, S., Su, C.Y. (2011). An analytical generalized Prandtl-Ishlinskii model inversion for hysteresis compensation in micropositioning control. IEEE/ASME Transactions on Mechatronics, vol. 16, no. 4, p. 734-744, DOI:10.1109/TMECH.2010.2052366.

[33] Li, Z., Feng, Y., Chai, T.Y., Fu, J., Su, C.Y. (2012). Modeling and compensation of asymmetric hysteresis nonlinearity for magnetostrictive actuators with an asymmetric shifted Prandtl-Ishlinskii model. Proceedings of the American Control Conference, p. 1658-1663.

[34] Gu, G.Y., Zhu, L.M., Su, C.Y. (2014). Modeling and compensation of asymmetric hysteresis nonlinearity for piezoceramic actuators with a modified Prandtl-Ishlinskii model. IEEE Transactions on Industrial Electronics, vol. 61, no. 3, p. 1583-1595, DOI:10.1109/TIE.2013.2257153.

[35] Jiang, F.L., Tao, G.L., Liu, H. (2015). Research on PMA properties and humanoid lower limb application. Proceedings of the IEEE International Conference on Advanced Intelligent Mechatronics, p. 1292-1297, D0I:10.1109/ AIM.2015.7222717.

[36] Chen, Y.S., Qiu, J.H., Sun, H. (2013). A hybrid model of Prandtl-Ishlinskii operator and neural network for hysteresis compensation in piezoelectric actuators. International Journal of Applied Electromagnetics and Mechanics, vol. 41, p. 335347, D0I:10.3233/JAE-131648.

[37] Aljanaideh, O., Rakheja, S., Su, C. Y. (2013). A PrandtlIshlinskii model for characterizing asymmetric and saturated hysteresis of smart material actuators. Intelligent Robotics and Applications, Lecture Notes in Computer Science, vol. 8103, p. 635-643, D0I:10.1007/978-3-642-40849-6_63.

[38] Jiang, H., Ji, H.L., Qiu, J.H., Chen, J.Q. (2010). A modified Prandtl-Ishlinskii model for modeling asymmetric hysteresis of piezoelectric actuators. IEEE Transactions on Ultrasonics, Ferroelectrics, and Frequency Control, vol. 57, no. 5, p. 12001210, DOI:10.1109/TUFFC.2010.1533. 